\title{
Practical Feasibility of Arago-Biot and Lorentz-Lorenz Theory Through Variation of Refractive Index of Typical Binary Liquid Mixtures Employing Low-Cost Experimental Setup
}

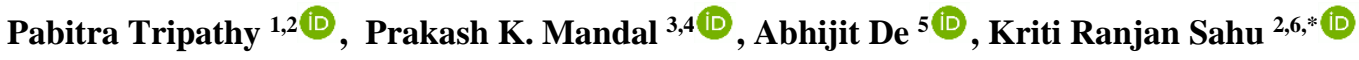 \\ Dept. of Physics, P K College, Contai 721401, Purba Medinipur, W. B., India; pabitraphysics001@ gmail.com (P.T.); \\ Dept. of Physics, Egra SSB College, Egra 721429, Purba Medinipur, W. B., India; kriti.sahu91@ gmail.com(K.R.S.); \\ Dept. of Chemistry, Egra SSB College, Egra, 721429, Purba Medinipur, W. B., India; prakash12chem@gmail.com \\ (P.K.M.); \\ 4 Dept. of Chemistry, University of Calcutta, 92, A. P. C. Road, Kolkata 700009, W. B., India \\ 5 Dept. of Physics, Taki Govt. College, Taki 743429, North 24-Paraganas., W. B., India; abhijit@tgc.ac.in (A.D.); \\ 6 Dept. of Physics, Bhatter College, Dantan 721426, Paschim Medinipur, W. B., India \\ * Correspondence: kriti.basis2020@gmail.com (K.R.S.);
}

Received: 25.04.2021; Revised: 3.06.2021; Accepted: 6.06.2021; Published: 13.08.2021

\begin{abstract}
Optical properties of the solutions comprising of two or more miscible liquids have been of immense interest both in the area of chemical and physical sciences. To date, there are reports on studies regarding different combinations of binary liquid mixtures. However, the experiments involved are either high-ended or using sophisticated instrumentation. Our prime objective is to set up a simple laboratory arrangement to estimate the refractive index of typical binary-liquid mixtures obtained by proportionate variations in combinations selecting from benzene, ethyl acetate, tetrahydrofuran, and water; without involving high-standard instrumentation or expensive laboratory setups. In the present study, we adopted a basic method to determine the refractive index of pure liquids of low polarity, like, benzene $\left(\mathrm{C}_{6} \mathrm{H}_{6}\right)$ and tetrahydrofuran or THF $\left(\mathrm{C}_{4} \mathrm{H}_{8} \mathrm{O}\right)$ and of high polarities, such as ethyl acetate or EtOAc or EA $\left(\mathrm{C}_{4} \mathrm{H}_{8} \mathrm{O}_{2}\right)$, and water $\left(\mathrm{H}_{2} \mathrm{O}\right)$ and also their binary homogeneous mixture with high accuracy. Our experimental data involving variation of refractive index with molar volume fraction matched very well with theoretical interpretations by Arago-Biot and Lorentz-Lorenz equation. In our results, density corrections have been neglected as we have chosen non-volatile solvents.
\end{abstract}

Keywords: refractive index; binary-liquid mixtures; Arago-Biot equation; Lorentz-Lorenz equation; benzene $\left(\mathrm{C}_{6} \mathrm{H}_{6}\right)$; tetrahydrofuran $\left(\mathrm{C}_{4} \mathrm{H}_{8} \mathrm{O}\right)$; ethyl acetate $\left(\mathrm{C}_{4} \mathrm{H}_{8} \mathrm{O}_{2}\right)$; low cost experimental setup.

(C) 2021 by the authors. This article is an open-access article distributed under the terms and conditions of the Creative Commons Attribution (CC BY) license (https://creativecommons.org/licenses/by/4.0/).

\section{Introduction}

Optical, translational, molecular, structural, and other important physical properties of liquid solutions, such as vapor pressure, surface tension, viscosity, ultrasonic velocity, volumetric and acoustic properties alongside refractive index, have been seeking attention or attracted the attention of physicists, physical chemists, and experimental theoreologists primarily of both academic and practical interest[1-30]. In most cases, the physical properties of pure liquid or mixture of liquids, such as dielectric constant, polarizability, density, mole fraction (for mixtures), etc., have been explored through measurement of refractive index at a different variation of concentration and temperature. On the one hand, polarization in optically active solution involving solute-solvent combinations led us to determine the concentration and 
percentage of solute in the solution using a polarimeter with required accessories. For this purpose, a simple kind of polarimeter setup can be used, which has opened up recent interest in this area. On the other hand, there has been constant effort to determine the refractive index of liquid solution to probe the properties of matter like dielectric permittivity, density, surface tension, and impurity in the liquid [31-35]. There are a host of refractometers with all types of advancement, but any experimental work that holds back is the laboratory setup space, cost factor, and procurement time. Considering these aspects, we adopted a basic and elementary method to estimate the refractive index of the solutions using the liquids easily available in an undergraduate and or high-school laboratory. After passing the incident laser beam through binary liquid mixtures, linear dispersion of refracted laser beam on a measurement scale was recorded. Such liquids were arbitrarily chosen considering their polarity, boiling point (b.p.), and miscibility of respective solvents poured in a hollow prism. Our study selected and used 4 combinations, namely, Benzene-EA, Benzene-THF, THF-EA, Water-THF of binary solutions of commonly available four liquids. Interestingly, we could observe that the experimental data collected from our method matched very well to the theoretical data for a homogeneous binary mixture of water and THF in a modified range to a volume fraction of $20 \%$ of water in THF only. However, in the case of other binary combinations of THF and benzene, benzene and ethyl acetate, and ethyl acetate and THF, respectively, some inconsistencies with theoretical data have been observed. We applied our experimental result to Arago-Biot and LorentzLorenz theory to understand its practical credibility. It is noteworthy to mention here that we have been successful in correlating the experimental data with theory. Thus, we could correlate the deviation of experimental data very well with theoretical data with our results. In our study, the density correction for evaporation of the mixture has been neglected as we have selected solvents having a boiling point much higher than room temperature.

\section{Theory}

It is well known fundamentally that when a beam of light falls on a prism, it suffers a minimum deviation depending on the type of medium of the prism and eventually undergoes dispersion to give either band spectrum or line spectra which depends on the type of source used. Usually, the light source is made variable by selecting either white light or discharge light sources having more than one wavelength component. For this purpose, spectrometer arrangements having collimator, prism bench, and telescope are used. On the other hand, if a fixed source comprising a monochromatic coherent light beam is incident on a glass prism, then a study can be performed if the medium of the prism is made variable. Further, an experimental setup could be constructed such that the transparent medium of the prism can be made variable.

In an attempt to vary the medium of an optical prism, a hollow glass prism having the shape and dimensions of a regular optical bench prism was fabricated locally [36]. The liquid optical medium, which was filled up in the prism, was selected from some commonly available chemical reagents, which were systematically mixed by varying their liquid composition by volume or molar fraction. In such an effort, a monochromatic light beam (Laser source) is made incident on the glass surface of the hollow optical prism surface (at point $\mathbf{Q}$ on $\mathbf{M N}$ surface, in Figure 1a) at an angle $\boldsymbol{i}$ '. The light beam is made to enter into the glass prism container and pass through the liquid medium of the prism, which is kept parallel to the prism's base. The light beam then comes out from the NO surface and gets refracted from point $\mathbf{R}$. The optical path (PQRS) is indicated by the arrow (in Figure 1a). The refracted angle is ' $\boldsymbol{i}$ '. In the absence 
of prism or presence of a hollow prism, the incident ray (PQ) goes straight forward, i.e., follow the PQTV path. The refracted ray (RS), in the presence of prism filled with the liquid, gets deviated from direct ray (PQTV) [in the absence of liquid inside the prism] forms an angle $\delta$, called deviation angle. When $\boldsymbol{i}=\boldsymbol{i}^{\prime}$, then $\boldsymbol{\delta}=\boldsymbol{\delta}_{\mathrm{m}}$, gives the minimum angle of deviation. The refractive index of the materials of the prism is $\mu$, defined at minimum deviation condition of the refracted light by equation by equation (1)

$$
\begin{gathered}
\mu=\frac{\sin \left(\frac{\varphi+\delta_{\mathbf{m}}}{2}\right)}{\sin \left(\frac{\varphi}{2}\right)} \ldots \ldots \ldots \\
\text { Where } \quad \delta_{\mathbf{m}}=\tan ^{-1}\left(\frac{\mathrm{vs}}{\mathrm{VT}}\right)
\end{gathered}
$$

and $\varphi$ is the angle of the prism.

The refractive index of a medium correlates well with the polarization of its molecules by the electromagnetic field of light [34]. For liquid mixtures, a number of mixing rules for the refractive index $(\mu)$ of a mixture in terms of the individual refractive indices $\left(\mu_{j}\right)$ of the pure components have been proposed. Amongst the liquid mixture rules, the simplest is a direct volume fraction average of the refractive index of each component $\left(\mu_{\mathrm{j}}\right)$, known as the Arago-Biot (A-B) equation (3) [34, 37]

$$
\mu=\sum_{j=1}^{n} Y_{j} \mu_{j} \ldots \ldots
$$

Where the nominal volume fraction $\mathrm{Y}_{\mathrm{j}}=\mathrm{V}_{\mathrm{j}} / \Sigma_{\mathrm{j}} \mathrm{V}_{\mathrm{j}}$, with $\mathrm{V}_{\mathrm{j}}$ denoting the volume of the $\mathrm{jth}$ component before mixing. Instead of the refractive index itself, the Newton equation (4) [34] takes the average of $\mu_{\mathrm{j}}^{2}$

$$
\mu^{2}=\sum_{j=1}^{n} Y_{j} \mu_{j}^{2} \text { where }, Y_{j}=\frac{X_{j} V_{j}}{\sum_{j=1}^{n} X_{j} V_{j}} \quad \ldots \quad \ldots \quad \ldots
$$

Further, Wiener, Heller, Gladstone-Dale (G-D), Lorentz-Lorenz (L-L), and few others proposed different equations for many possible binary liquid mixtures. However, amongst them, the most systematic mixing rule is the Lorentz-Lorenz equation [34]. The L-L equation is fundamentally based on an argument that led to the Clausius - Mossotti equation for the dielectric constant of polarizable molecules, which assumes ideal mixing of the polarizability. The Lorentz-Lorenz (L-L) equation (5) reads

$$
\frac{\mu^{2}-1}{\mu^{2}+2}=\sum_{j=1}^{n} Y_{j} \frac{\mu_{j}^{2}-1}{\mu_{j}^{2}+2} \quad \ldots \quad \ldots \quad \ldots
$$

Where $Y_{j}=$ volume fraction of liquid used in mixture, $X_{j}=$ mole fraction and $V_{j}=$ molar volume of component $\mathrm{j}, \mathrm{j}=2$ for binary mixture and $\mathrm{j}=3$ for ternary mixture.

The parameters were experimentally measured and calculated to obtain the value of the refractive index $(\mu)$ of the single or mixture liquid. 

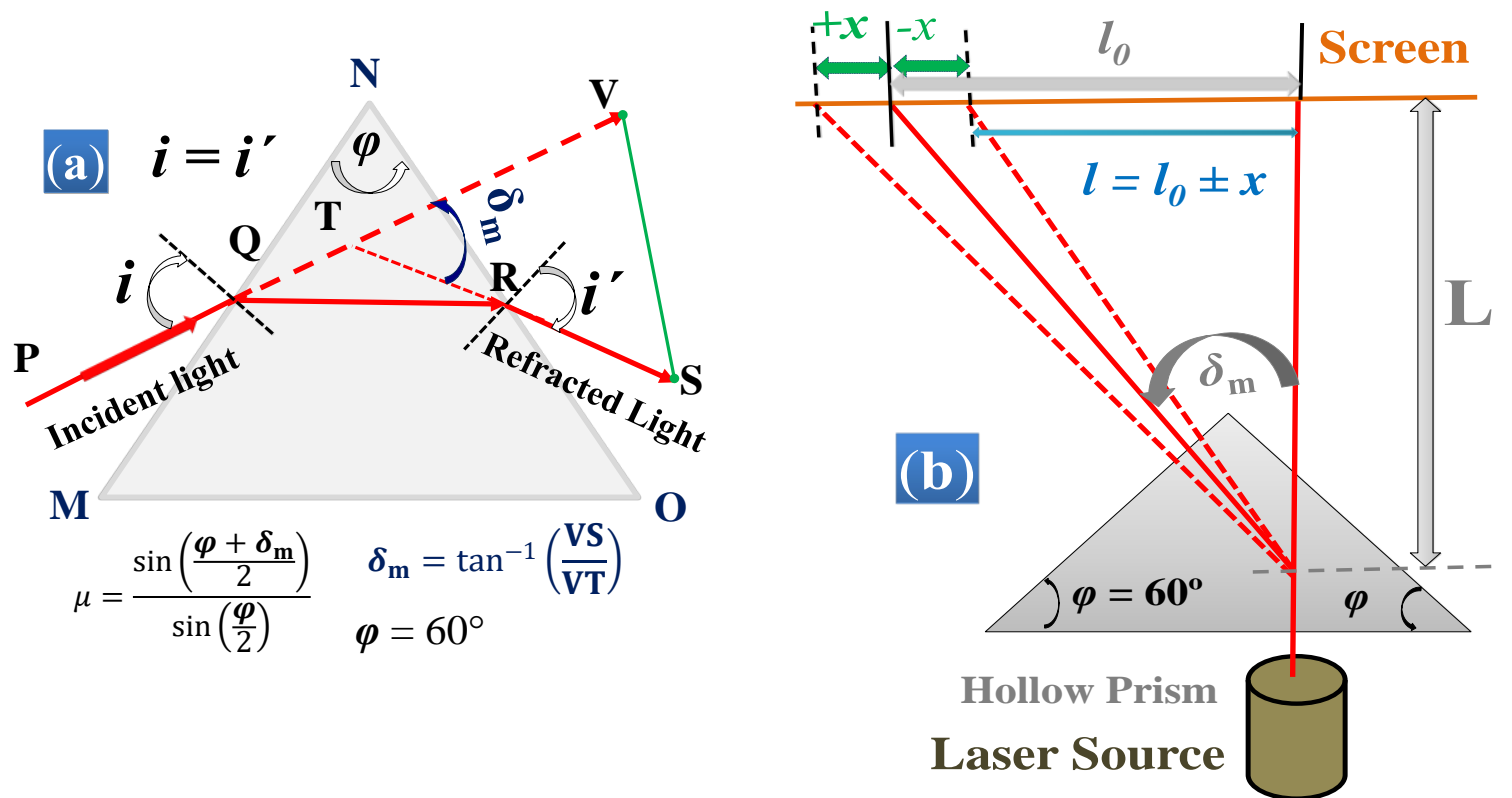

Figure 1.Schematic diagram of (a) Optical path of the incident light, transmitted and refracted light passing through a prism, and (b) Experimental setup for our study.

\section{Materials and Methods}

\subsection{Chemicals.}

Ethyl acetate (EA) $\left(\mathrm{C}_{4} \mathrm{H}_{8} \mathrm{O}_{2}\right)$ [Extra Pure, M.W. of 88.11 g.mole ${ }^{-1}$, density (at $20^{\circ} \mathrm{C}$ ) of 0.90 g.cm ${ }^{-3}$, Mfg-SPL, India]; Benzene $\left(\mathrm{C}_{6} \mathrm{H}_{6}\right)$ [Purity G.C. $\geq 99 \%$, M.W. of 78.11 g.mole ${ }^{-1}$, density (at $20^{\circ} \mathrm{C}$ ) of $0.878 \mathrm{~g} \cdot \mathrm{cm}^{-3}$, Mfg-Merck SPL, India] and Tetrahydrofuran (THF) $\left(\mathrm{C}_{4} \mathrm{H}_{8} \mathrm{O}\right)$ [Purity G.C. $\geq 99 \%$, M. W. of 72.11 g.mol ${ }^{-1}$, density (at $20^{\circ} \mathrm{C}$ ) of 0.889 g.cm ${ }^{-3}$, Mfg-Merck SPL, India] were used to prepare binary liquid mixtures.

\subsection{Measurements.}

3.2.1. The apparatus used for the present experimentation - Simple and Low-Cost Laboratory Setup.

The main components constituting our experimental setup were the following - a thermometer of the range $\left(0\right.$ to $\left.200{ }^{\circ} \mathrm{C}\right)$, a hollow glass prism of dimension $6.35 \mathrm{~cm}$ made from a glass sheet of thickness $\sim 2.75 \mathrm{~mm}$ and the angle of the prism, $\varphi=60^{\circ}$, with a glass made lid of same thickness having a very small sized hole in the center, a laser source (wavelength of $632 \mathrm{~nm}$ ), measuring cylinder (for measurement of the volume of liquids), regular meter scale, normal stand and clamp arrangement, graph paper fitted on the laboratory wall (screen), and glass rod as a stirrer. The separation between prism and screen was $\mathbf{L}($ about $465 \mathrm{~cm}$ ). The laser source was positioned at about $6 \mathrm{~cm}$ apart from the prism. The prism was fabricated such that a maximum volume of about $160 \mathrm{ml}$ could be accommodated.

\subsubsection{Method adopted for determination of Refractive Index.}

We used the accessories available in any common undergraduate laboratory and employed some of the simplest methods of measurement to determine the refractive indices of liquid mixtures; viz, namely using (i) a Plane mirror and a convex lens, (ii) Rectangular hollow block, (iii) Hollow prism. 
In this study, a hollow prism was fabricated to measure the refractive indices of binary liquid mixtures. We employed the method where the refractive index of the water liquid mixture has been determined by illuminating a hollow prism by filling in a different molar ratio in terms of volume fraction(v\%). For this purpose, a laser ray of wavelength $632 \mathrm{~nm}$ was made incident on our fabricated prism, and a simple arrangement was made to measure the minimum deviation angle $\left(\boldsymbol{\delta}_{\mathbf{m}}\right)$ of a laser beam. When the prism, with angle $\boldsymbol{\varphi}$ is placed at minimum deviation position, the refractive index ( $\boldsymbol{\mu})$ was obtained using the equation (1). We can obtain the expression for minimum deviation angle $\left(\boldsymbol{\delta}_{\mathbf{m}}\right)$; equation (6) as (from Figure $1 \mathrm{~b}$ ),

$$
\boldsymbol{\delta}_{\boldsymbol{m}}=\tan ^{-1}\left(\frac{\boldsymbol{l}}{\boldsymbol{L}}\right)=\tan ^{-1}\left(\frac{\boldsymbol{l}_{\mathbf{0}} \mp \boldsymbol{x}}{\boldsymbol{L}}\right) \ldots \ldots \ldots
$$

Here, $\mathbf{L}$ is the distance between the screen and the prism, $\boldsymbol{l}_{\mathbf{0}}$ is the linear deflection of the laser spot on the screen. Next, $\boldsymbol{l}$ can be found by either subtracting and or adding $\boldsymbol{x}$ from $\boldsymbol{l}_{\mathbf{0}}$, where $\boldsymbol{l}_{\mathbf{0}}$ is the distance between the starting point of the scale and the position of the undeviated laser spot. The position of the laser spot on the scale-graph position is designated by ' $x$ '.

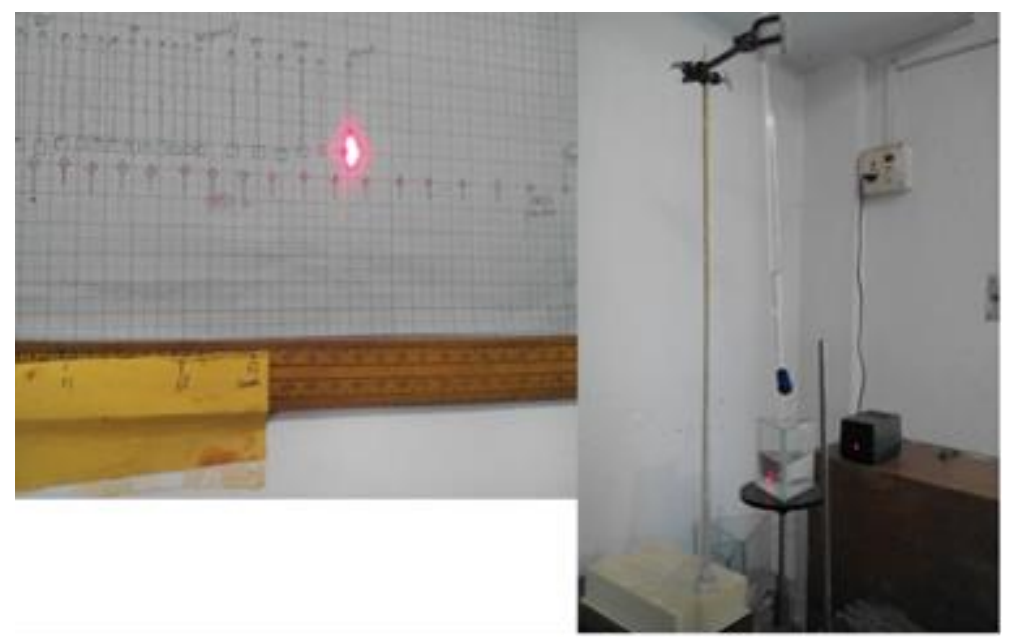

Figure 2. Photograph showing the actual experimental arrangement to measure the parameters and calculate the value of the refractive index $(\boldsymbol{\mu})$ as a function of the minimum angle of deviation using a laser source of the single or liquid mixture.

\section{Results and Discussion}

At the very outset of this article, we have chosen four solvents viz. benzene and THF as non-polar and moderately polar solvent along with water and ethyl acetate (EA) as polar solvent. All liquids are colorless and have higher than room temperature boiling-point (b.p) temperatures, e.g., b.p. of benzene, THF, water and ethyl acetate (EA) are $80.1{ }^{\circ} \mathrm{C}, 66.0^{\circ} \mathrm{C}$, $100.0^{\circ} \mathrm{C}, 77.1^{\circ} \mathrm{C}$; respectively,[38] which was needed and formed the basis for our experiment. We all know one of the most popular phrases in solvent chemistry "like dissolves like". This is mainly applicable to solutes along with their suitable solvent for complete dissolution, but it can also be applied in case of solvent miscibility. [39, 40] Solvent miscibility or solute dissolution depends on van der Waals force of attraction [41-61] between non-polar entities and dipole-dipole interaction [62-73] between polar entities. H-bonding [62, 74-77] is the strongest dipolar interaction among weak chemical forces of attraction and in the case of polar molecules having high electronegative atoms like fluorine, oxygen, and nitrogen. On the other 
hand, theoretical perspectives London force [63, 55-61, 78-86] operates between two non-polar molecules maintaining an optimum distance amongst them. London force is nothing but a kind of van der Waals force of attraction generated by inducing dipole on a non-polar molecule by another adjacent non-polar molecule having instantaneous dipole that would be created in it in terms of disturbance in symmetry of electrical charge into the molecule. It is noteworthy to mention here that London force is applicable in very short-range distances, inversely proportional to the seventh power of internuclear distance of two molecules at close proximity. [58-61]This force is a low energetic phenomenon. It is true that the momentary dipoles and induced dipoles change continuously to maintain the neutral state. However, the attractive force so generated constantly binds the molecules together. London force or van der Waals force of attraction increases with an increase in Molecular Weight [M. W.] and surface area of a nonpolar substance; but whenever the distance between two molecules becomes less than van der Waals radii, they repulse each other, and it is known as van der Waals repulsion. The dipoledipole interaction and London force are $\sim 8 \mathrm{~kJ} \mathrm{~mol}^{-1}$ and $\sim 4 \mathrm{~kJ} \cdot \mathrm{mol}^{-1}$ [61]; respectively. Polarity depends on structure and bond dipole moments which are directly related to the electronegativity difference between two atoms attached through that bond. Benzene has a planar hexagonal symmetrical structure, and all bonds are between two carbon atoms (sp2 hybridized); so that there is no bond moment in any bond, and that's why benzene has no resultant dipole moment. So, it has zero molecular dipole moment and is treated as a non-polar liquid. THF has a pentagonal alicyclic structure, and one oxygen atom is incorporated in the saturated five-membered ring system having two $\mathrm{C}-\mathrm{O}$ bonds and eight $\mathrm{C}-\mathrm{H}$ bonds. Hence, it has some bond moments for $\mathrm{C}-\mathrm{O}$ bonds and $\mathrm{C}-\mathrm{H}$ bonds. THF shows an overall dipole moment of $1.63 \mathrm{D}$. Water has a bent structure with two $\mathrm{O}-\mathrm{H}$ bonds having a high bond dipole moment due to the large electronegativity difference between the two elements. For bent structure, the bond moments do not cancel out each other mutually; rather exerts a high molecular dipole moment value of $1.85 \mathrm{D}$ due to the resultant vector sum of two bond moments. Ethyl acetate has a zigzag structure as it is an aliphatic compound comprising three $\mathrm{sp} 3$ carbons and one sp2 carbon with two oxygen atoms. In the structure of ethyl acetate, a $\mathrm{C}=\mathrm{O}$, two $\mathrm{C}-\mathrm{O}$ and eight $\mathrm{C}$ $\mathrm{H}$ bonds contribute individual bond moment value to overall dipole moment value of $1.78 \mathrm{D}$. Experimentally established refractive index data for benzene, THF, water and Ethyl acetate are $1.50,1.41,1.33$, and 1.37 at $20^{\circ} \mathrm{C}$.

In our present interest, as benzene is immiscible in water due to the huge polarity difference, this combination for binary mixture is not considered. However, benzene is miscible with THF and EA by inducing dipole-dipole interaction between a non-polar and a polar liquid. THF gets miscible into the water through H-bonding. THF and Ethyl Acetate, both being polar aprotic liquids, are miscible through simple dipole-dipole interaction. In addition, Ethyl Acetate and water binary mixture become too turbid, and so, that was not considered suitable for our experiment. We, therefore, selected the other four binary combinations from these four liquids for our purpose, viz., (i) Benzene and Ethyl Acetate; (ii) Benzene and THF; (iii) Water and THF; (iv) THF and Ethyl Acetate. On the basis of the miscibility and solubility factors of benzene, ethyl acetate, and THF we selected the binary liquid mixtures accordingly; to conduct our optical experiments [86-90].

Table 1. Structure and related properties of benzene, THF, water and ethyl acetate $[38,91,92]$. 
Structure

(in 3D ball-stick)
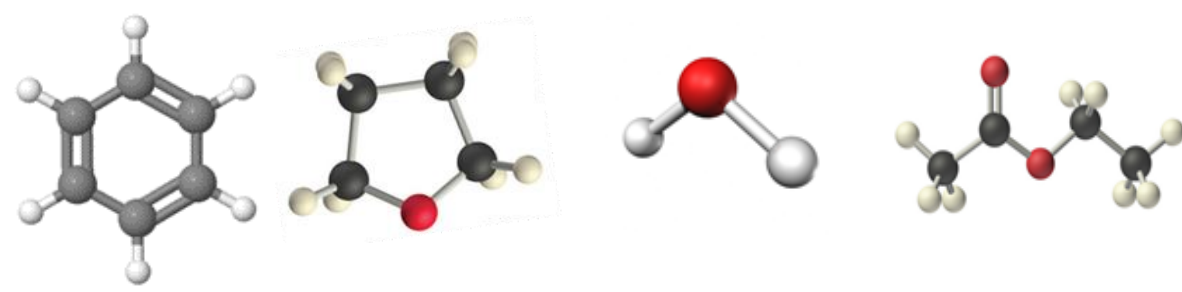

Structure (in 2D)With the
direction of molecular dipole
moment

The interesting point of our study is concerning the nature of variation of $\Delta \mu$ for (THF + water) with volume fraction of water which is in analogy with a variation of $\Delta \mu$ for other binary liquid mixture combinations involving benzene, EA, and THF. Moreover, variations observed in our case for benzene, EA, and THF agree with variations of refractive index as a function of the mole fraction of acetonitrile in a binary mixture of water and acetonitrile without salt [34].

Dubey et al. (2005) reported a detailed study of the refractive index of ternary liquid mixtures involving - Squalane, Cyclohexane, hexane, and benzene using Abbe Refractometer at different temperature settings [35]. They categorically reported a good theoretical experimental matching of data using A-B and L-L relations. Further, they concluded that L-L and A-B were accepted as the best theoretical mixing rules behaving in a simple quantitative manner and performed well within the experimental error limits. This simply means that our experimental data's good match and fitting to A-B and L-L theoretical relations are in coherence and well accepted.

It is evident from Figure 3, that the selection of the laser (wavelength of $632 \mathrm{~nm}$ ) used in our experiment resulted in a linear relation between refractive index and minimum angle of deviation. This indicates an application to calibration for selecting binary liquid mixture medium offering required variation in refractive index as a function of the minimum angle of deviation.

In Figure 4, a binary liquid mixture of benzene (B) and Ethyl Acetate (EA) was considered for variation of refractive index $(\mu)$ versus volume fraction of EA (YEA). The figure shows that as the volume fraction of EA increases from 0.0 (pure benzene) to 0.6 the estimated refractive index decreased exponentially from 1.47 to almost 1.40 . This implies clearly that the 
optical density of the binary liquid mixture decreased accordingly. Moreover, the calculated values of refractive indices from both $\mathrm{A}-\mathrm{B}$ and $\mathrm{L}-\mathrm{L}$ relation supported the decrease in the refractive index; but the decrease was rather linear. Figure 5 refers to the variation of $\Delta \mu(=\mu \exp$ $-\mu_{\text {theo }}$ ) as a function of volume fraction of EA. In this figure, the $\Delta \mu$ or difference in refractive index obtained from experimental $\left(\mu_{\exp }\right)$ and theoretical $\left(\mu_{\text {theo }}\right)$ values attains a maximum value of 0.025 at about a value of 0.125 of YEA. Figure 6 , indicated a difference of $\Delta \mu$ of magnitude 0.075 with the addition of about $60 \%$ EA, without molar calculations.

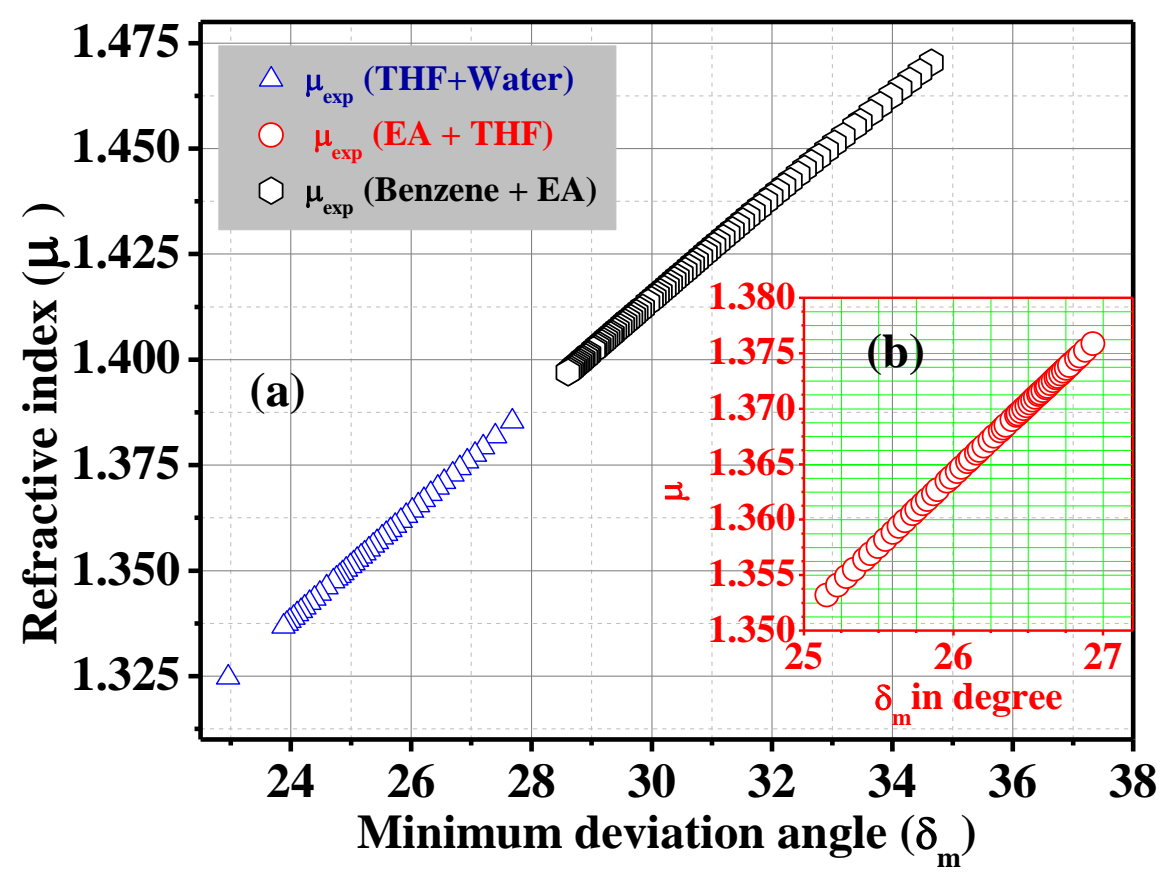

Figure 3. Variation of minimum deviation angle $\left(\delta_{\mathrm{m}}\right.$ in degree or $\left.{ }^{\circ}\right)$ with refractive index $(\mu)$ of binary liquid mixture selected from benzene, ethyl acetate (EA), THF, and Water.

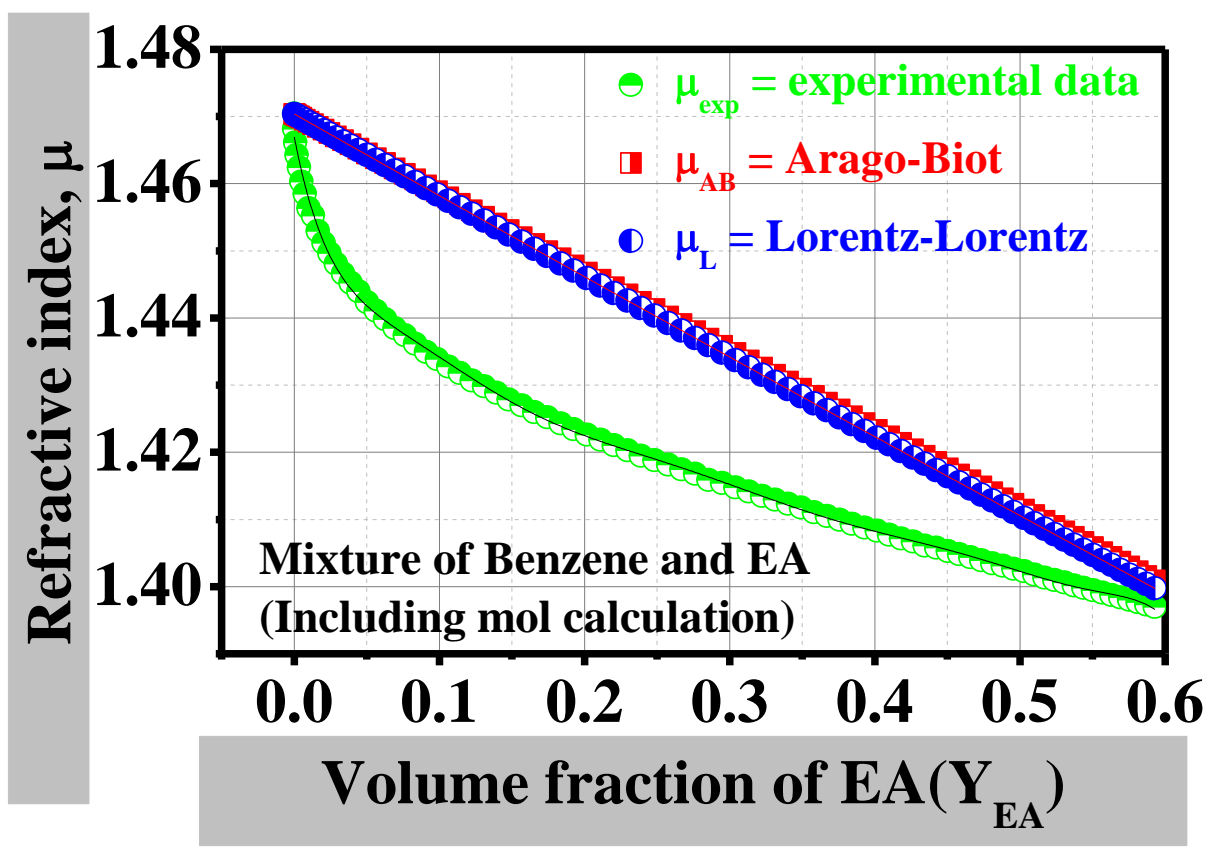

Figure 4. Refractive index $(\mu)$ of benzene and its mixture with different volume mole fractions of EA (ethyl acetate) up to $60 \%$. 


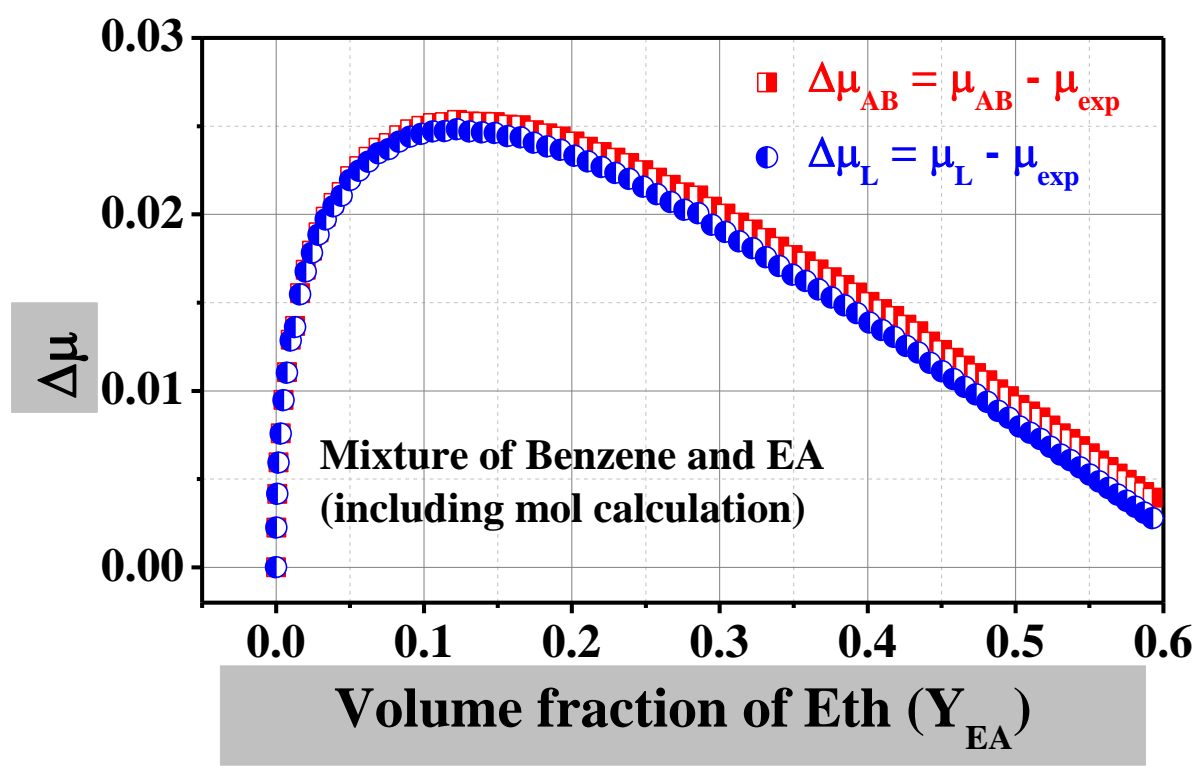

Figure 5. Deviation of refractive indices of experimental data from theoretical data for different mole fractions of EA (ethyl acetate) with benzene.

In Figure 7, a binary liquid mixture of benzene (Ben) and Tetrahydrofuran (THF) was considered for variation of refractive index $(\mu)$ versus volume mole fraction of Benzene ( $Y_{\text {Ben }}$ ). At the beginning, i.e., for pure THF the value of the refractive index was 1.385, which increased almost linearly to a value of 1.46 for the binary liquid mixture of THF and Benzene. In both cases, A-B and L-L, the theoretical values of the refractive index increased linearly with an increase in benzene content in the liquid mixture. A slight deviation in the variation of $\mu \approx 0.02$ was observed at $Y_{B e n}$ of 0.1 . Figure 8, depicted a perfect match between experimental data and theoretical (A-B) and (L-L) values. At $\mathrm{Y}_{\mathrm{Ben}}$ of 0.15 the maximum value of $\Delta \mu$ is about 0.015 . Further, for higher values of $Y_{\text {Ben }}$ a mono-atomic decrease of $\Delta \mu$ is observed. Figure 9 , shows almost a linear increase of refractive index from 1.385 (for pure THF) to 1.455 (for the binary liquid mixture of benzene and THF) which was at $\mathrm{Y}_{\mathrm{Ben}}=0.7$.

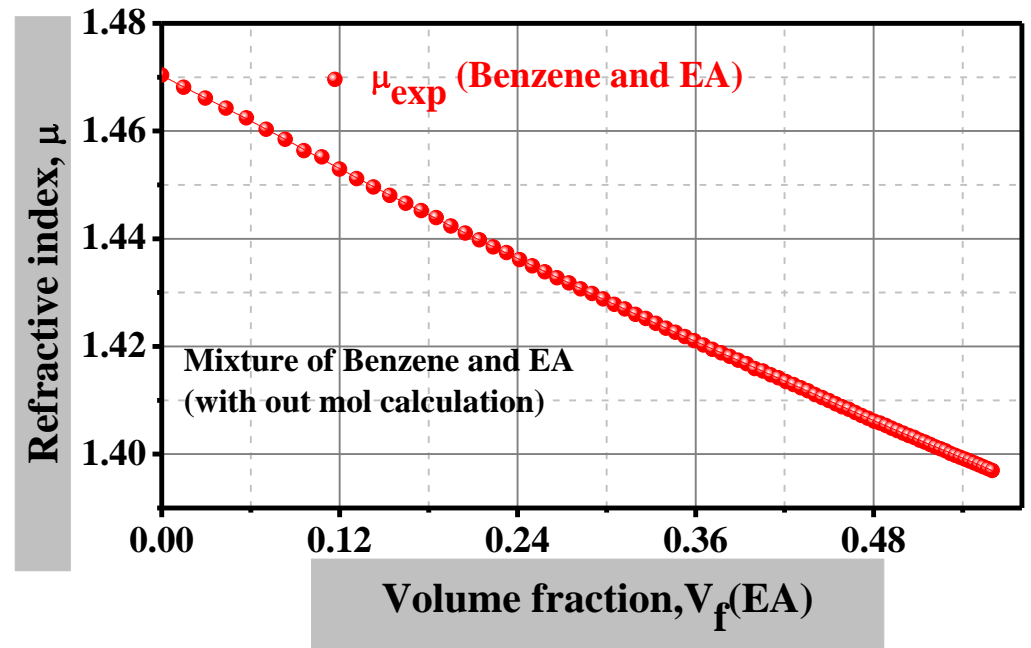

Figure 6. Refractive index $(\mu)$ of benzene and its mixture with different volume fractions (without mole calculation) of ethyl acetate (EA) upto $60 \%$. 


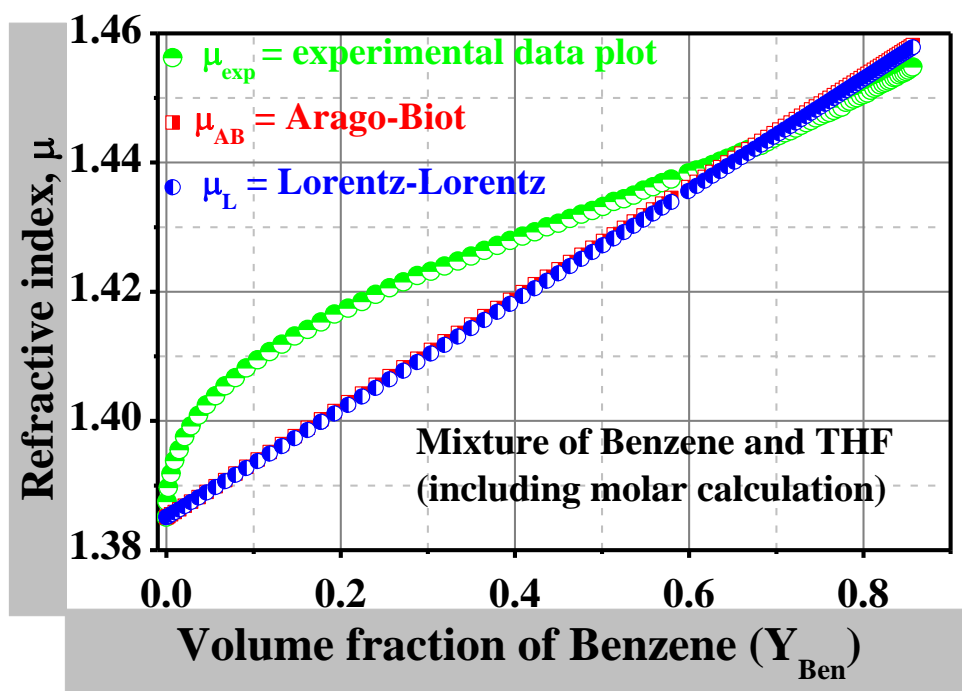

Figure 7. Refractive index $(\mu)$ of $\mathrm{THF}$ and its mixture with different volume mole fractions of benzene above $90 \%$.

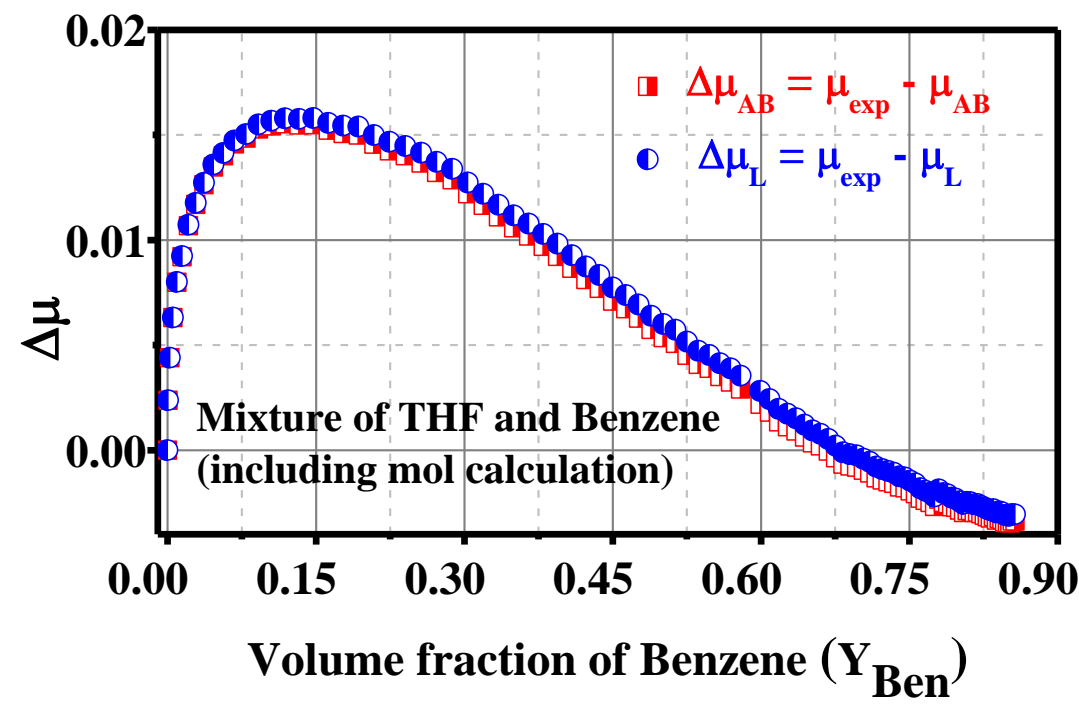

Figure 8. Deviation of refractive indices of experimental data from theoretical data for different mole fractions of benzene with THF.

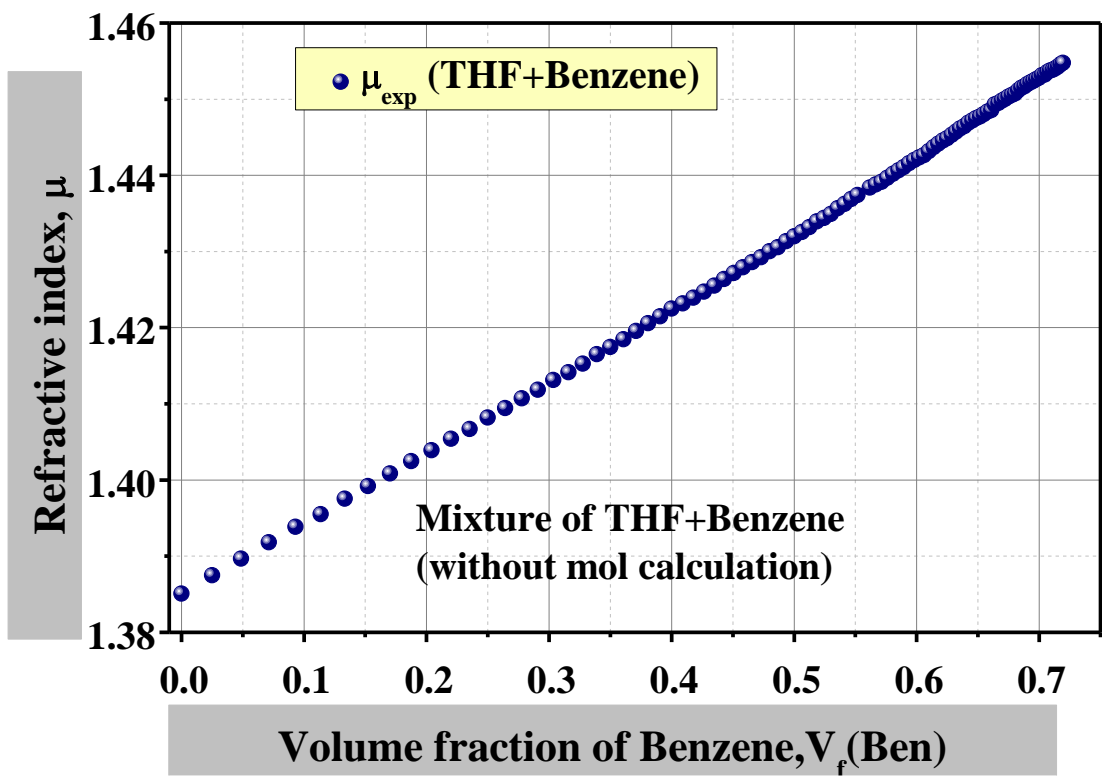

Figure 9. Refractive index ( $\mu$ ) of THF and its mixture with different volume fractions (without mole calculation) of benzene above $90 \%$. 
In Figure 10, a binary liquid mixture of ethyl acetate (EA) and Tetrahydrofuran (THF) was considered where the value of the refractive index increased from 1.355 (for pure EA) to 1.38 (for the binary mixture $\mathrm{EA}+\mathrm{THF}$ ) as $\mathrm{Y}_{\mathrm{THF}}$ increased from 0.0 to a value of 0.85 . Figure 11, shows a variation of $\Delta \mu$ versus mole fraction of THF, which is very similar to a binary liquid mixture of (Ben + THF) for both experimental and theoretical values (A-B and (L-L) of refractive index. In this figure, the maximum value of $\Delta \mu$ is 0.004 at $\mathrm{Y}_{\mathrm{THF}}$ value of 0.1 . Further, the value of $\Delta \mu$ decreased as $\mathrm{Y}_{\mathrm{THF}}$ attained a value of 0.5 .

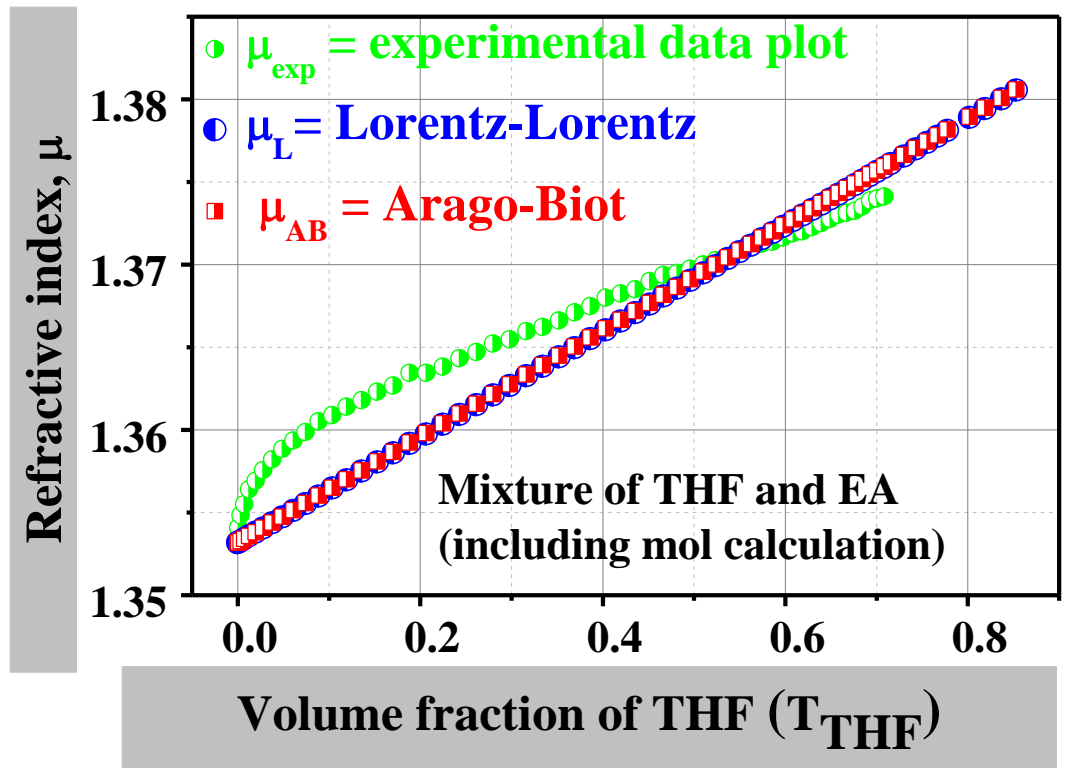

Figure 10. Refractive index $(\mu)$ of THF and its mixture with different volume mole fraction of ethyl acetate up to $80 \%$.

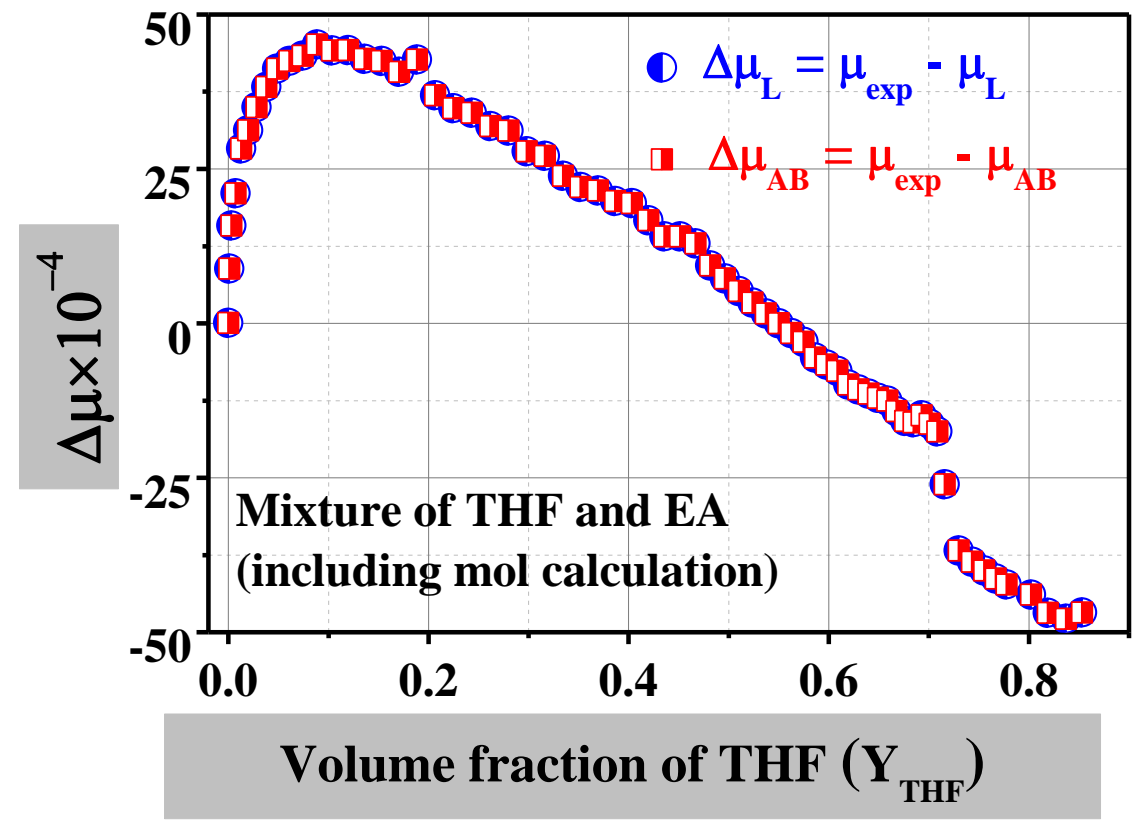

Figure 11. Deviation of refractive indices of experimental data from theoretical data for different mole fractions of THF with ethyl acetate. 


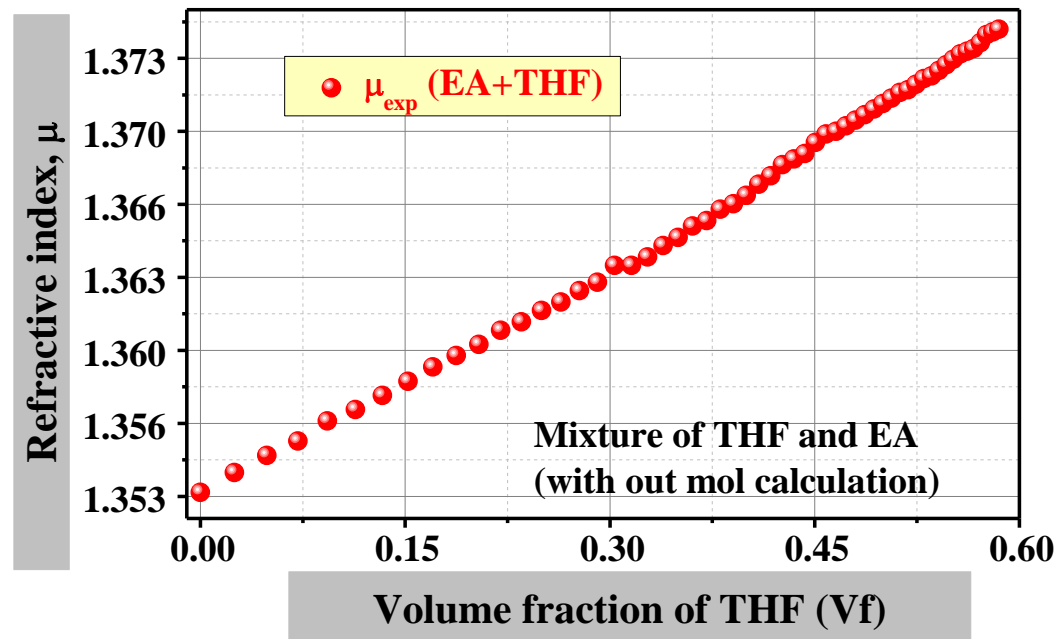

Figure 12. Refractive index ( $\mu$ ) of THF and its mixture with different volume fractions (without mole calculation) of ethyl acetate up to $60 \%$.

Figure 13, refers to a binary liquid mixture of THF with water. In this figure, the refractive index of pure THF $(\mu=1.385)$ decreased almost linearly to 1.335 (pure water) as THF got subsequently and sufficiently diluted with water. Further, in Figure 14, a maximum value of $\Delta \mu$ for this binary liquid mixture, of (THF + water), attained a value of 0.015 as the volume fraction of water ( $\mathrm{Y}_{\text {water }}$ ) increased to a value of about 0.8. Moreover, all the experimental data for the binary liquid mixture was in total agreed with A-B and L-L calculated values.

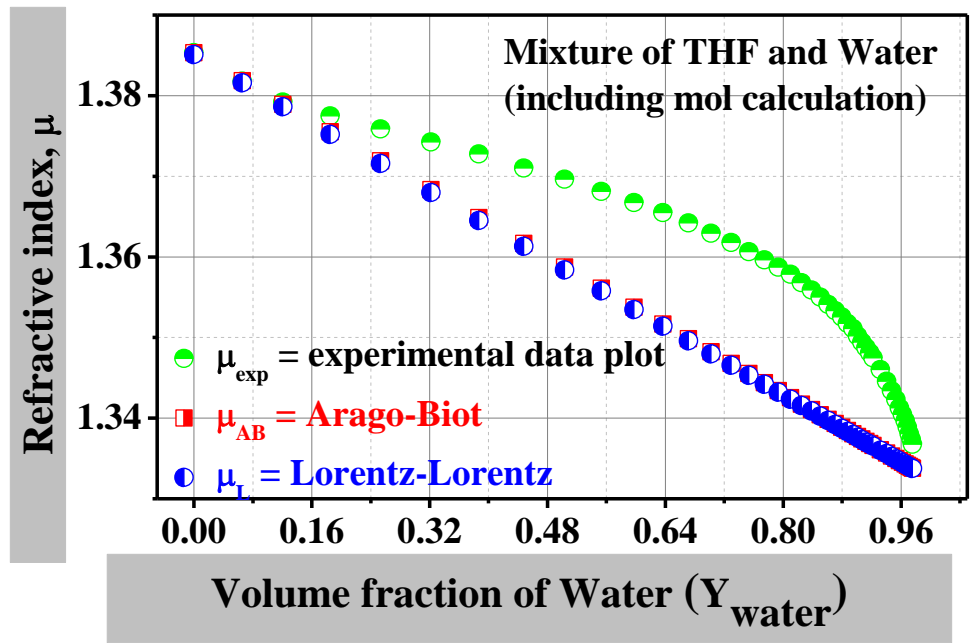

Figure 13. Refractive index $(\mu)$ of THF and its mixture with different volume mole fractions of water up to $98 \%$. 


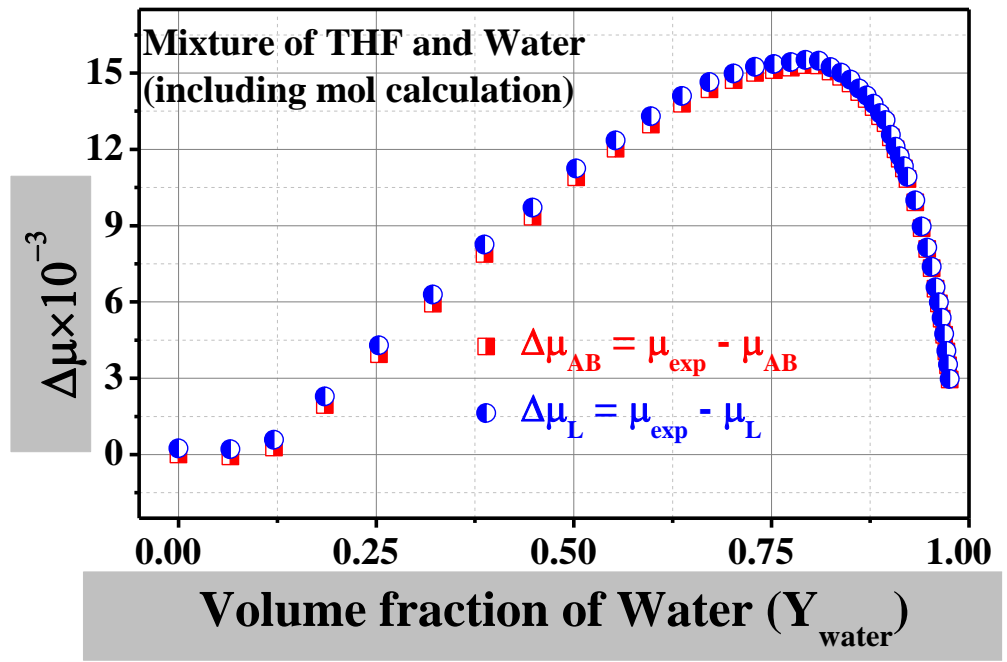

Figure 14. Deviation of refractive indices of experimental data from theoretical data for different mole fractions of THF with Water.

This section may be divided into subheadings. It should provide a concise and precise description of the experimental results, their interpretation, and the experimental conclusions that can be drawn.

\section{Conclusions}

We could set up a very basic and simple experimental arrangement using very common - low-cost laboratory components. Using this facility, we systematically measured the refractive index of uniform binary liquid mixtures, like, benzene, ethyl acetate, THF, and water with very high accuracy. Our selection of the binary liquid mixtures involving benzene, EA, THF, and water were based on their miscibility and solubility factors. The nature of variation of $\Delta \mu$ for (THF + water) with volume fraction of water is observed analogous to the variation observed for $\Delta \mu$ as a function of volume fraction for other binary liquid mixture combinations involving benzene, EA, and THF. The experimental data fit well with the theoretical data in the lower range (volume mole fraction of $20 \%$ of water with THF) only for a uniform binary mixture of water and THF but differed for other binary mixtures, like (THF and benzene) (benzene and ethyl acetate) and (ethyl acetate and THF). The deviation of experimental data/curve ( $\mu$ vs. molar volume fraction) from theoretical data/curve (Arago-Biot and Lorentz-Lorenz equation) has been observed is in good agreement with our results. The linear relation observed for the refractive index as a function of minimum deviation appears to be a promising calibration factor. To our delight, by sharing, that we have been successful at our very first attempt with this setup. Further, we very much hope that this experiment will open up many routes of modern research concerning the study of different inherent properties of solo, binary or ternary liquid mixture employing an economical and basic laboratory setup.

\section{Funding}

Self-funding.

\section{Acknowledgments}

We are thankful to Mr. Jyotindranath Mirdda (Naraharipur Primary School), Dr. Pradipta Panchadhyayee (P. K. College, Contai), who provided constant encouragement to carry out the 
project work. Thanks to the authority of Egra S S B College, Egra and P. K. College, Contai, and also thanks to all authors of all references.

\section{Conflicts of Interest}

The authors declare no conflict of interest.

\section{References}

1. Matsubara, H.; Chiguchi, K.; Law, B.M. Pickering Emulsion Transitions in 2,6-Lutidine Plus Water Critical Liquid Mixtures. Langmuir 2020, 36, 12601-12606, https://pubs.acs.org/doi/10.1021/acs.langmuir.0c02161.

2. Havasi, D.; Farkas, D.; Mika, L.T. Isobaric Vapor-Liquid Equilibria of Binary Mixtures of $\gamma$-Valerolactone + Acetone and Ethyl Acetate. J. Chemical \& Engineering Data 2020, 65, 419-425, https://doi.org/10.1021/acs.jced.9b00379.

3. Seki, A.; Yoshio, M.; Mori, Y.; Funahashi, M. Ferroelectric Liquid-Crystalline Binary Mixtures Based on Achiral and Chiral Trifluoromethylphenylterthiophenes. ACS Applied Materials \& Interfaces 2020, 12, 53029-53038, https://doi.org/10.1021/acsami.0c17717.

4. Hoga, H.E.; Olivieri, G.V.; Torres, R.B. Experimental Measurements of Volumetric and Acoustic Properties of Binary Mixtures of 1-Butyl-3-methylimidazolium Hexafluorophosphate with Molecular Solvents. $J$. Chemical \& Engineering Data 2020, 65, 3406-3419, https://pubs.acs.org/doi/10.1021/acs.jced.9b01032.

5. Gilani, A.G.;Dafrazi, A.A. Systematic Study on Physicochemical and Related Excess Properties of Several Binary Systems of Cyclic Ketones and Alkanediols (C2-C5). J. Chemical \& Engineering Data 2020, 65, 4, 1886-1899, https://pubs.acs.org/doi/10.1021/acs.jced.9b01100.

6. Rocky, M.M.H.; Akhtar, S. Correlations and Predictions for Viscosity of Binary Liquid Systems: New UNIFAC-VISCO Interaction Parameters for $\mathrm{O}, \mathrm{N}$, and S Containing Organic Liquids. Industrial \& Engineering Chemistry Research 2020, 59, 8004-8017, https://pubs.acs.org/doi/10.1021/acs.iecr.0c00335.

7. Azarang, N.; Movagharnejad, K.; Pirdashti, M.; Ketabi, M. Densities, Viscosities, and Refractive Indices of Poly(ethylene glycol) $300+$ 1,2-Ethanediol, 1,2-Propanediol, 1,3-Propanediol, 1,3-Butanediol, or 1,4Butanediol Binary Liquid Mixtures. J. Chemical \& Engineering Data 2020, 65, 3448-3462, https://pubs.acs.org/doi/10.1021/acs.jced.9b01189.

8. Chaudhary, N.; Nain, A.K. Densities, Ultrasonic Speeds, Viscosities, Refractive Indices, and Excess Properties of 1-Butyl-3-methylimidazolium Tetrafluoroborate + N-Methylacetamide Binary Mixtures at Different Temperatures. J. Chemical \& Engineering Data 2020, 65, 1447-1459, https://pubs.acs.org/doi/10.1021/acs.jced.9b00875.

9. Ouaar, F.; Mokbel, I.; Negadi, A.; Aguilar, F.; Montero, E. A.; Jose, J.; Bahadur, I.; Negadi, L. Vapor-Liquid Equilibria, Density, Sound Velocity, and Refractive Index for Binary Mixtures Containing 2-(2Ethoxyethoxy)ethanol and 1-Propanol or 2-Propanol or 1-Butanol or 2-Butanol at Different Temperatures. $J$. Chemical \& Engineering Data 2020, 65, 2351-2372, https://pubs.acs.org./doi/10.1021/acs.jced.9b00959.

10. Abroodi, M.; Bagheri, A.;Razavizadeh, B.M. Surface Tension of Binary and Ternary Systems Containing Monoethanolamine (MEA), Water and Alcohols (Methanol, Ethanol, and Isopropanol) at $303.15 \mathrm{~K} . J$. Chemical \& Engineering Data 2020, 65, 3173-3182, https://pubs.acs.org/doi/10.1021/acs.jced.0c00192.

11. Wagh, P.S.; Bhumkar, A.N.;Rathnam, M.V. Excess Volume, Viscosity, and Isentropic Compressibility of Methyl Acrylate + Alkane Binary Mixtures. J. Chemical \& Engineering Data 2020, 65, 5, 2343-2350, https://pubs.acs.org/doi/10.1021/acs.jced.9b00924.

12. Pasham, F.; Jabbari, M.; Farajtabar, A. Solvatochromic Measurement of KAT Parameters and Modeling Preferential Solvation in Green Potential Binary Mixtures of N-Formylmorpholine with Water, Alcohols, and Ethyl Acetate. J. Chemical \& Engineering Data 2020, 65, 5458-5466, https://pubs.acs.org/doi/10.1021/acs.jced.0c00589.

13. Zhang, L.; Zhang, H.; Kwon, Y.M.; Shahzad, N.; Chen, H.; Wang, X.; Liu, A.; Zhang, L.; Zhu, D.; Xia, X.; Gao, D.; Kim, C.K. Combined Experimental and Theoretical Studies on the Prediction of the Isobaric VaporLiquid Association Phenomena for Binary and Ternary Mixtures of Water, Ethanoic Acid, and Propanoic Acid. Industrial \& Engineering Chemistry Research 2020, 59, 13290-13304, https://pubs.acs.org/doi/10.1021/acs.iecr.0c01963. 
14. Fan, J.; Meng, Q.; Song, F. Experimental Research on the Thermal Conductivity of Binary Mixtures with a Biodiesel Compound Ethyl Laurate + Three Alcohols (1-Propanol, 1-Butanol, and 1-Pentanol). J. Chemical \& Engineering Data 2020, 65, 4649-4655, https://pubs.acs.org/doi/10.1021/acs.jced.0c00505.

15. Troni, K.L.; Damaceno, D.S.;Ceriani, R. Evaluation of a Variation of the Differential Scanning Calorimetry Technique for Measuring Boiling Points of Binary Mixtures at Subatmospheric Pressures. J. Chemical \& Engineering Data 2020, 65, 3334-3343, https://pubs.acs.org/doi/10.1021/acs.jced.0c00111.

16. Pikkarainen, L.Viscosities of binary solvent mixtures of N,N-diethylmethanesulfonamide with aliphatic alcohols. Journal of Chemical \& Engineering Data 1988, 33, 299-301, https://doi.org/10.1021/je00053a022.

17. Qiu, J.; Albrecht, J.; Janey, J.Synergistic Solvation Effects: Enhanced Compound Solubility Using Binary Solvent Mixtures. Organic Process Research \& Development2019, 23, 1343-1351, https://doi.org/10.1021/acs.oprd.9b00077.

18. Dahlberg, M.; Laaksonen, A.Preferential Solvation of Phenol in Binary Solvent Mixtures. A Molecular Dynamics Study. The J. Physical Chemistry A 2006, 110, 2253-2258, https://doi.org/10.1021/jp056463e.

19. Srivastava, A.K.; Shankar, S.L.Ionic Conductivity in Binary Solvent Mixtures. 4. Dimethyl Sulfoxide + water at $25^{\circ} \mathrm{C}$. Journal of Chemical \& Engineering Data 2000, 45, 92-96, https://doi.org/10.1021/je990185t.

20. Zhang, J.; Huang, C.; Xu, R.Solubility of Bifonazole in Four Binary Solvent Mixtures: Experimental Measurement and Thermodynamic Modeling. J. Chemical \& Engineering Data 2019, 64, 2641-2648, https://doi.org/10.1021/acs.jced.9b00097.

21. Liu, X.; Li, W.; He, Y.; Du, S.; Yin, H.; Gong, J.; Wu, S.Solubility Measurement and Correlation of Ceftiofur Sodium Trihydrate in Four Binary Solvent Mixtures. Journal of Chemical \& Engineering Data 2020, 65, 2, 916-922, https://doi.org/10.1021/acs.jced.9b01088.

22. Heric, E.L.; Posey, C.D. Refractive Indices of Some Ternary Systems Containing Naphthalene. Binary Solvent Mixtures Containing Hexadecane. J. Chemical \& Engineering Data 1965, 10, 340-343, https://doi.org/10.1021/je60027a011.

23. Gupta, S.Solvation chemistry through synergism: static and dynamic features of n-amyl alcohol-chloroform binary solvent mixture. RSC Adv. 2016, 6, 99306-99313, https://doi.org/10.1039/C6RA23231K.

24. Li,X.; Chen,J.; Chen, G.; Zhao, H.Solubility modelling, solution thermodynamics and preferential solvation of hymecromone in binary solvent mixtures of N,N-dimethylformamide + methanol, ethanol or n-propanol. RSC Adv. 2017, 7, 46378-46387, https://doi.org/10.1039/C7RA07587A.

25. Ahmadi,R.; Hemmateenejad,B.; Safavi,A.; Shojaeifard,Z.; Shahsavar,A.; Mohajeri,A.; Dokoohaki, H. M.; Zolghadr, A.R.Deep eutectic-water binary solvent associations investigated by vibrational spectroscopy and chemometrics. Phys. Chem. Chem. Phys. 2018, 20, 18463-18473, https://doi.org/10.1039/C8CP00409A.

26. Ràfols,C.; Rosés, M.; Bosch, E.Solute-solvent and solvent-solvent interactions in binary solvent mixtures. Part 5. Preferential solvation of solvatochromic indicators in mixtures of propan-2-ol with hexane, benzene, ethanol and methanol. J. Chem. Soc., Perkin Trans. 1997, 2, 243-248, https://doi.org/10.1039/A605421H.

27. Bosch, E.; Rosés, M.Relationship between ET polarity and composition in binary solvent mixtures. J. Chem. Soc. Faraday Trans. 1992, 88, 3541-3546,https://doi.org/10.1039/FT9928803541.

28. Cabot, R.; Hunter, C.A.A thermodynamic study of selective solvation in solvent mixtures. Org. Biomol. Chem. 2010, 8, 1943-1950, https://doi.org/10.1039/B927107D.

29. Angulo, G.; Brucka, M.; Gerecke, M.; Grampp, G.; Jeannerat, D.; Milkiewicz, J.; Mitrev, Y.; Radzewicz, C.; Rosspeintner, A.; Vauthey, E.; Wnuk, P.Characterization of dimethylsulfoxide/glycerol mixtures: a binary solvent system for the study of "friction-dependent" chemical reactivity. Phys. Chem. Chem. Phys. 2016, 18, 18460-18469, https://doi.org/10.1039/C6CP02997C.

30. Herodes, K.; Leito, I.; Koppel, I.; Rosés, M.Solute-solvent and solvent-solvent interactions in binary solvent mixtures. Part 8. The ET(30) polarity of binary mixtures of formamides with hydroxylic solvents. J. Phys. Org. Chem. 1999, 12, 109-115, https://doi.org/10.1002/(SICI)1099-1395(199902)12:2<109::AIDPOC97>3.0.CO;2-L.

31. Newton, I. Opticks: or, A treatise of the reflections, refractions, inflexions and colours of light; Printed for Sam. Smith, and Benj. Walford, London, 1704, https://doi.org/10.5479/sil.302475.39088000644674.

32. Malecki, J.; Dutkiewicz, M. Nonlinear Dielectric Effect Study of Tetrahydrofuran Association in Benzene and Cyclohexane Solutions. J. Solution Chem. 1999, 28, 101-116, https://doi.org/10.1023/A:1021793928300.

33. Heller, W. Remarks on Refractive Index Mixture Rules. J. Phys. Chem. 1965, 69, 1123-1129, https://doi.org/10.1021/j100888a006. 
34. An, N.; Zhuang, B.; Li, M.; Lu, Y.; Wang, Z.G. Combined Theoretical and Experimental Study of Refractive Indices of Water-Acetonitrile-Salt Systems. J. Phys. Chem. B. 2015, 119, 10701-10709, https://doi.org/10.1021/acs.jpcb.5b05433.

35. Dubey G.P.; Tripathi N.; Bhatia S.C. Refractive Index of Ternary Liquid Systems of Squalane (+hexane + benzene), Squalane (+ Cyclohexane + benzene) and Squalane (+hexane + Cyclohexane). Ind. J. Pure \& Applied Physics. 2005, 43, 175-179.

36. Astrua, M.; Pisami, M. Prism refractive index measurement at INRiM. Meas. Sci. Technol. 2009, 20, 095305, https://doi.org/10.1088/0957-0233/20/9/095305.

37. Gayathri, A.; Venugopal, T.; Padmanaban, R.; Venkatramanan, K.; Vijayalakshmi, R. A comparative study of experimental and theoretical refractive index of binary liquid mixtures using mathematical methods.IOP Conf. Series: Materials Science and Engineering 2018, 390, 012116, https://doi.org/10.1088/1757899X/390/1/012116.

38. Smallwood, I.M. Handbook of Organic Solvent properties, $1^{\text {st }}$ Edition, Arnold, London, UK, 1996.

39. Marcus, Y. The Properties of Solvents, Wiley Series in Solution Chemistry, Volume $-4,1^{\text {st }}$ Edition, Wiley, Chichester, UK, 1998, $44-49$.

40. Wypych, G. Handbook of Solvents, $1^{\text {st }}$ Edition, Chem Tech Publishing, Toronto, Canada, 2001, 7 - 58, 130 $-131$.

41. Everett, D.H.; Richmond, P. The theory and calculation of van der waals forces.Colloid Science. 1975, 2 , 130-172, https://doi.org/10.1039/9781847555847-00130.

42. Black, W.; de Jongh, J.G.V.; Overbeek, J.Th.G.; Sparnaay, M.J. Measurements of retarded Van Der Waals' forces. Trans. Faraday Soc.1960, 56, 1597-1608, https://doi.org/10.1039/TF9605601597.

43. Rücker, A.W.XXII.-On the range of molecular forces.J. Chem. Soc. Trans. 1888, 53, 222-262, https://doi.org/10.1039/CT8885300222.

44. MacKerell Jr., A.D.; Karplus, M.Importance of Attractive van der Waals Contribution in Empirical Energy Function Models for the Heat of Vaporization of Polar Liquids. The J. Physical Chemistry 1991, 95, 1055910560, https://pubs.acs.org/doi/10.1021/j100179a013.

45. Drummond, C.J.; Chan, D.Y.C. van der Waals Interaction, Surface Free Energies, andContact Angles: Dispersive Polymers and Liquids. Langmuir 1997, 13, 3890-3895, https://pubs.acs.org/doi/10.1021/la962131c.

46. Malrieu, J.P. High Orders Corrections to the Van der Waals-London Forces. II. Interaction of Two Molecules with Isotropic Polarizabilities. Int. J. Quantum Chemistry 1971, 5, 455-467, https://doi.org/10.1002/qua.560050408.

47. Dryden, D.M.; Hopkins, J.C.; Denoyer, L.K.; Poudel, L.; Steinmetz, N.F.; Ching, W.Y.; Podgornik, R.; Parsegian, A.; French, R.H. van der Waals Interactions at the Mesoscale: Open-ScienceImplementation, Anisotropy, Retardation, and Solvent Effects. Langmuir 2015, 31, 10145-10153, https://doi.org/10.1021/acs.langmuir.5b00106.

48. Nerenberg, P.S.; Jo, B.; So, C.; Tripathy, A.; Gordon, T.H. Optimizing Solute-Water van der Waals Interactions To ReproduceSolvation Free Energies. The J. Physical Chemistry B 2012, 116, 4524-4534, https://pubs.acs.org/doi/10.1021/jp2118373.

49. Lin, I.C.; Seitsonen, A.P.; Coutinho-Neto, M.D.; Tavernelli, I.; Rothlisberger, U.Importance of van der Waals Interactions in Liquid Water. The J. Physical Chemistry B 2009, 113, 1127-1131, https://pubs.acs.org/doi/10.1021/jp806376e.

50. Ricceri, R.; Romeu, N. V.; Taddei, G. van der Waals Interactions in Nonionic Micelles. Langmuir 1996, 12, 913-915, https://pubs.acs.org/doi/10.1021/la950609r.

51. Han, Z.; Wei, X.; Xu, C.; Chiang, C.-1.;Zhang, Y.; Ho, W. Imaging van der Waals Interactions. The J. Physical Chemistry Letters 2016, 7, 5205-5211, https://doi.org/10.1021/acs.jpclett.6b02749.

52. Käfer, D.; Helou, M. E.; Gemel, C.; Witte, G.Packing of Planar Organic Molecules: Interplay of van der Waalsand Electrostatic Interaction.Crystal Growth \& Design 2008, 8, 3053-3057, https://doi.org/10.1021/cg800195u.

53. Maeda, H.; Maeda, Y.Orientation-Dependent London-van der Waals Interaction Energybetween Macroscopic Bodies. Langmuir 2015, 31, 7251-7263, https://doi.org/10.1021/acs.langmuir.5b01459.

54. Ewing, G. E. Intermolecular Interactions: van der Waals Molecules. Angewandte Chemie International Edition in English 1972, 11, 6, 486-495, https://doi.org/10.1002/anie.197204861. 
55. Pollice, R.; Bot, M.; Kobylianskii, I. J.; Shenderovich, I.; Chen, P. Attenuation of London Dispersion in Dichloromethane Solutions. J. American Chemical Society. 2017, 139, 13126-13140, https://doi.org/10.1021/jacs.7b06997.

56. Prieve, D. C.; Ruckenstein, E. Effect of London forces upon the rate of deposition of Brownian particles. AlChE Journal. 1974, 20, 1178-1187, https://doi.org/10.1002/aic.690200618.

57. Malrieu, J. P. High orders corrections to the Van der Waals-London forces. II. Interaction of two molecules with isotropic polarizabilities. J. Quantum Chemistry, 1971, 5, 455-467, https://doi.org/10.1002/qua.560050408.

58. Hahn, P.S.; Chen, J.D.; Slattery, J. C. Effects of London van der Waals forces on the thinning and rupture of a dimpled liquid film as a small drop or bubble approaches a fluid fluid interface, AIChE J. 1985, 31, 20262038, https://doi.org/10.1002/aic.690311212.

59. London, F. The general theory of molecular forces. Trans. Faraday Soc. 1937, 33, 8b-26, https://doi.org/10.1039/TF937330008B.

60 Imbihl, R. van der Waals London forces. In Catalysis from A to Z. 2021, https://doi.org/10.1002/9783527809080.cataz17736.

61. Masliyah, J.H; Bhattacharjee, S. London-Van Der Waals Forces and the Dlvo Theory. $1^{\text {st }}$ Eds., John Wiley \& Sons, Hoboken, New Jersey, 2005, 391-425, Chapter. 11, Inc., https://doi.org/10.1002/0471799742.ch11.

62. Gao, X.C.; Hao, Q.; Wang, C.S.Improved Polarizable Dipole-dipole Interaction Model for Hydrogen Bonding, Stacking, T-shaped and X-H· $\pi$ Interactions. J. Chemical Theory and Computation 2017, 13, 27302741, https://doi.org/10.1021/acs.jctc.6b00936.

63. Shibata, M.; Kuntzleman, T.S. Intermolecular Interactions: Dipole-Dipole, Dipole-Induced Dipole, and London Dispersion Forces. J. Chemical Education 2009, 86, 1469, https://doi.org/10.1021/ed086p1469.1.

64. Haskell, R.W.Thermodynamic model of dipole-dipole and dipole-induced dipole interactions in polar mixtures. The Journal of Physical Chemistry 1969, 73, 2916-2919, https://doi.org/10.1021/j100843a022.

65. Yoder, C.H. Teaching ion-ion, ion-dipole, and dipole-dipole interactions. J. Chemical Education 1977, 54, 402, https://doi.org/10.1021/ed054p402.

66. Urbakh, M.; Klafter, J. Dipole-Dipole Interactions near Interfaces. The J. Physical Chemistry 1993, 97, 33443349, https://doi.org/10.1021/j100115a042.

67. Nicholls, L.J. A demonstration of dipole-dipole interactions.J. Chemical Education 1983, 60, 993, https://doi.org/10.1021/ed060p993.

68. Dereka, B.; Vauthey, E. Solute-Solvent Interactions and Excited-State Symmetry Breaking:Beyond the Dipole-Dipole and the Hydrogen-Bond Interactions.The J. Physical Chemistry Letters 2017, 8, 3927-3932, https://doi.org/10.1021/acs.jpclett.7b01821.

69. Abraham, R.J. Medium Effects in Rotational Isomerism. VI. Inclusion of Dipole-Dipole Interactions in Polar Solvents. The Journal of Physical Chemistry 1969, 73, 1192-1199, https://doi.org/10.1021/j100725a003.

70. Xu, L.; Miao, X.; Ying, X.; Deng, W.Two-Dimensional Self-Assembled Molecular Structures Formedby the Competition of van der Waals Forces and Dipole-DipoleInteractions. The J. Physical Chemistry C 2012, 116, 1061-1069, https://doi.org/10.1021/jp210000e.

71. Li, S.S.; Huang, C.Y.; Hao, J.J.; Wang, C.S. A polarizable dipole-dipole interaction model for evaluation of the interaction energies for $\mathrm{N}-\mathrm{H} \bullet \bullet \mathrm{O}=\mathrm{C}$ and $\mathrm{C}-\mathrm{H} \bullet \bullet \mathrm{O}=\mathrm{C}$ hydrogen-bonded complexes. J. Computational Chemistry 2013, 35, 415-426, https://doi.org/10.1002/jcc.23473.

72. Claverie, P.; Rein, R. Theory of intermolecular interactions: The long range terms in the dipole-dipole, monopoles-dipole, and monopoles-bond polarizabilities approximations. Int. J. Quantum Chemistry 1969, 3, 537-551, https://doi.org/10.1002/qua.560030502.

73. Knežević, G.; Marinković, M.; TošIć, B. On some specific properties of systems endowed with dipole-dipole interactions. Int. J. Quantum Chemistry 1977, 12, 785-794, https://doi.org/10.1002/qua.560120502.

74. di Nunzio, M. R.; Wang, Y.; Douhal, A. Structural spectroscopy and dynamics of inter- and intramolecular H-bonding interactions of topotecan, a potent anticancer drug, in organic solvents and in aqueous solution. The J. Physical Chemistry B 2012, 116, 7522-7530, https://pubs.acs.org/doi/10.1021/jp302923a.

75. Marvel, C.S.; Harkema, J.; Copley, M.J. Hydrogen Bonds Involving the C-H Link. XIV. ' Solubility of Donor Solutes in Hydrogen Bonding Solvents. J. American Chemical Society 1941,63, 1609, https://pubs.acs.org/doi/10.1021/ja01851a033.

76. Copley, M.J.; Zellhoefer, G.F.; Marvel, C.S. Hydrogen Bonds Involving the C-H Link. IV. The Effect of Solvent Association on Solubility. J. American Chemical Society 1938,60, 2666-2673, https://pubs.acs.org/doi/10.1021/ja01278a037. 
77. Malik,P.K.; Tripathy,M.; Kajjam, A.B.; Patel, S.Preferential solvation of p-nitroaniline in a binary mixture of chloroform and hydrogen bond acceptor solvents: the role of specific solute-solvent hydrogen bonding. Phys. Chem. Chem. Phys. 2020, 22, 3545-3562, https://doi.org/10.1039/c9cp05772b.

78. Earles, T. T. Can London Dispersion Forces Be Stronger than Dipole-Dipole Forces, Including Hydrogen Bonds?. Journal of Chemical Education. 1995, 72, 727, https://pubs.acs.org/doi/10.1021/ed072p727.

79. Ruckenstein, E.; Prieve, D.C. Rate of Deposition of Brownian Particles under the Action of London and Double-layer Forces. J. Chem. Soc., Faraday Trans. 2, 1973, 69, 1522-1536, https://doi.org/10.1039/F29736901522.

80. Hildebrand, J.H.; Rotariu, G. Some Applications of Solubility Theory to Analytical Problems. Analytical Chemistry 1952, 24,770-773, https://doi.org/10.1021/ac60065a002.

81. Grunwald, E.; Lipnick, R.L.; Ralph III, E.K. Lifetimes of Amine-Water and Amine-Alcohol HydrogenBonded Complexes in Hydroxylic Solvents. Role of London Dispersion Forces in Solvation. J. American Chemical Society 1969, 91,4333-4338, https://pubs.acs.org/doi/10.1021/ja01044a001.

82. Noyes, K.; Cooper, M.M. Investigating Student Understanding of London Dispersion Forces:A Longitudinal Study. J. Chemical Education 2019, 96, 1821-1832, https://pubs.acs.org/doi/10.1021/acs.jchemed.9b00455.

83. Homer, J.; Mohammadi, M.S. Polyatomic London Dispersion Forces. J. Chem. Soc., Faraday Trans.2, 1987, 83, 1957-1974, https://doi.org/10.1039/F29878301957.

84. Theresa Julia Zielinski. Fostering Creativity and Learning Using Instructional Symbolic Mathematics Documents. J Chemical Education 2009, 86, 1466-1469, https://pubs.acs.org/doi/10.1021/ed086p1469.1.

85. Chakrabarti, R.; Vekilov, P.G. Attraction between Permanent Dipoles and London Dispersion Forces Dominate the Thermodynamics of Organic Crystallization. Crystal Growth \& Design 2020, 20, 7429-7438, https://doi.org/10.1021/acs.cgd.0c01102.

86. Ghosh, S.K. Advanced General Organic Chemistry A ModernApproachPart I, $3^{\text {rd }}$ Edition, NCBA, Kolkata, India, 2009, $226-227$.

87. Solvent miscibility table. Available online: https://www.csustan.edu/sites/default/files/groups/Chemistry/Drake/documents/solvent_miscibility_table.p df.

88. O’Neil, M.J. (ed) The Merck Index - An Encyclopedia of Chemicals, Drugs, and Biologicals, Whitehouse Station, N.J.: Merck and Co., Inc. 2006, 1585.

89. Lide, D.R.; G.W.A. Milne (Eds.) Handbook of Data on Organic Compounds. volume I, 3rd Ed. CRC Press, Inc. Boca Raton, F.L., 1994.

90. Riddick, J.A.; Bunger, W.B.; Sakano, T.K. Techniques of Chemistry 4th Ed. Vol. II, Organic Solvents, N.Y.: John Wiley \& Sons., 1985, 309.

91. Properties of Solvents Chart | Sigma-Aldrich. Available online: Properties of Solvents Chart | Sigma-Aldrich (accessed on 18.01.2021).

92. Properties of Solvents Used in Organic Chemistry. Available online: Properties of Solvents Used in Organic Chemistry (murov.info) (accessed on 18.01.2021). 